\title{
Microarray-based screening of differentially expressed genes in glucocorticoid-induced avascular necrosis
}

\author{
GANGYONG HUANG* ${ }^{*}$ YIBING WEI*, GUANGLEI ZHAO, JUN XIA, SIQUN WANG, \\ JIANGUO WU, FEIYAN CHEN, JIE CHEN and JINGSHEN SHI
}

Department of Orthopaedics, Huashan Hospital, Fudan University, Shanghai 200040, P.R. China

Received January 20, 2016; Accepted February 14, 2017

DOI: $10.3892 / \mathrm{mmr} .2017 .6438$

\begin{abstract}
The underlying mechanisms of glucocorticoid (GC)-induced avascular necrosis of the femoral head (ANFH) have yet to be fully understood, in particular the mechanisms associated with the change of gene expression pattern. The present study aimed to identify key genes with a differential expression pattern in GC-induced ANFH. E-MEXP-2751 microarray data were downloaded from the ArrayExpress database. Differentially expressed genes (DEGs) were identified in 5 femoral head samples of steroid-induced ANFH rats compared with 5 placebo-treated rat samples. Gene Ontology (GO) and pathway enrichment analyses were performed upon these DEGs. A total 93 DEGs (46 upregulated and 47 downregulated genes) were identified in GC-induced ANFH samples. These DEGs were enriched in different GO terms and pathways, including chondrocyte differentiation and detection of chemical stimuli. The enrichment map revealed that skeletal system development was interconnected with several other GO terms by gene overlap. The literature mined network analysis revealed that 5 upregulated genes were associated with femoral necrosis, including parathyroid hormone receptor 1 (PTHR1), vitamin D (1,25-Dihydroxyvitamin D3) receptor $(V D R)$, collagen, type II, $\alpha 1$, proprotein convertase subtilisin/kexin type 6 and zinc finger protein 354C (ZFP354C). In addition, ZFP354C and VDR were identified to transcription factors. Furthermore, $P T H R 1$ was revealed to interact with $V D R$, and $\alpha$-2-macroglobulin $(A 2 M)$ interacted with fibronectin $1(F N I)$ in the PPI network. PTHRI may be involved in GC-induced ANFH via interacting with $V D R$. $A 2 M$ may also be involved in the development of GC-induced
\end{abstract}

Correspondence to: Dr Siqun Wang, Department of Orthopaedics, Huashan Hospital, Fudan University, 12 Wulumuqi Middle Road, Shanghai 200040, P.R. China

E-mail: cormierhuang@msn.com

*Contributed equally

Key words: vascular necrosis of the femoral head, differentially expressed genes, enrichment map, text-mining association network, protein-protein interaction network
ANFH through interacting with FN1. An improved understanding of the molecular mechanisms underlying GC-induced ANFH may provide novel targets for diagnostics and therapeutic treatment.

\section{Introduction}

Avascular necrosis of the femoral head (ANFH) is a progressive pathological process that leads to avascular necrosis and collapse of the femoral head due to an obstructed blood supply, resulting in impaired hip joint function and permanent disability $(1,2)$. ANFH causes significant morbidity as well as impairment of daily function to patients (3). In addition, ANFH is considered irreversible, therefore any diagnostic strategy or therapeutic intervention for ANFH is best introduced at an early stage (4).

Glucocorticoid (GC) administration is known to be the most common non-traumatic cause of ANFH (5). GCs are widely used to treat most inflammatory disorders and are also included in most chemotherapy protocols (6). Therefore, ANFH due to $\mathrm{GC}$ use is a potential major complication for a large patient group (6). However, the exact pathogenesis of AFNH associated with GC remains to be fully elucidated. There are several alternative mechanisms including fat embolization, modified artery constriction, intramedullary pressure changes, coagulation disorders, circulatory impairment and cell dysfunction. There is evidence that apoptosis of osteoblasts and osteocytes, not compromised vascularity, is the direct effect and primary etiology of GC-induced ANFH $(7,8)$. Kerachian et al (9). suggested that GCs may contribute to differential gene expression in ANFH in rats, and revealed that GC-induced ANFH in rats may be mediated by $\alpha-2$-macroglobulin $(A 2 M)$. In addition, Samara et al (10). demonstrated that osteoprotegerin, receptor activator of nuclear factor- $\mathrm{kB}$ (RANK) and RANK ligand mRNA levels were higher in the necrotic part of the femoral head of patients with avascular necrosis compared with the normal site (9). However, despite the strong association of GC with ANFH, the underlying mechanisms are yet to be fully understood, in particular the mechanisms associated with the change of gene expression pattern.

Using the same microarray data as Kerachian et al (9), the present study performed bioinformatic analysis with the aim of identifying additional genes associated with GC-induced ANFH. In the present study, differentially expressed genes (DEGs) were 
Table I. The enriched GO terms of upregulated genes and downregulated genes.

\begin{tabular}{|c|c|c|c|}
\hline Category & Term & Count & P-value \\
\hline \multicolumn{4}{|c|}{ Upregulated } \\
\hline \multirow[t]{8}{*}{$\mathrm{BP}$} & GO:0051216 cartilage development & 6 & $2.27 \times 10^{-06}$ \\
\hline & GO:0001501 skeletal system development & 8 & $1.06 \times 10^{-05}$ \\
\hline & GO:0006953 acute-phase response & 4 & $1.24 \times 10^{-04}$ \\
\hline & GO:0060348 bone development & 5 & $4.81 \times 10^{-04}$ \\
\hline & GO:0002062 chondrocyte differentiation & 3 & 0.00175 \\
\hline & GO:0030198 extracellular matrix organization & 4 & 0.0022 \\
\hline & GO:0002526 acute inflammatory response & 4 & 0.0024 \\
\hline & GO:0006954 inflammatory response & 5 & 0.0038 \\
\hline \multirow[t]{7}{*}{$\mathrm{CC}$} & GO:0005576 extracellular region & 17 & $7.61 \times 10^{-08}$ \\
\hline & GO:0044421 extracellular region part & 13 & $2.06 \times 10^{-07}$ \\
\hline & GO:0031012 extracellular matrix & 8 & $5.73 \times 10^{-06}$ \\
\hline & GO:0005615 extracellular space & 9 & $5.55 \times 10^{-05}$ \\
\hline & GO:0005578 proteinaceous extracellular matrix & 6 & $3.73 \times 10^{-04}$ \\
\hline & GO:0005604 basement membrane & 3 & 0.0181 \\
\hline & GO:0044420 extracellular matrix part & 3 & 0.0315 \\
\hline \multirow[t]{6}{*}{ MF } & GO:0005539 glycosaminoglycan binding & 5 & $1.49 \times 10^{-04}$ \\
\hline & GO:0001871 pattern binding & 5 & $2.45 \times 10^{-04}$ \\
\hline & GO:0030247 polysaccharide binding & 5 & $2.45 \times 10^{-04}$ \\
\hline & GO:0030246 carbohydrate binding & 6 & 0.0019 \\
\hline & GO:0005201 extracellular matrix structural constituent & 3 & 0.0037 \\
\hline & GO:0008201 heparin binding & 3 & 0.0158 \\
\hline \multicolumn{4}{|c|}{ Downregulated } \\
\hline \multirow[t]{8}{*}{$\mathrm{BP}$} & $\begin{array}{l}\text { GO:0007186 G-protein coupled receptor } \\
\text { protein signaling pathway }\end{array}$ & 33 & $8.15 \times 10^{-19}$ \\
\hline & GO:0007166 cell surface receptor linked signal transduction & 35 & $2.64 \times 10^{-18}$ \\
\hline & GO:0007606 sensory perception of chemical stimulus & 26 & $1.89 \times 10^{-15}$ \\
\hline & GO:0007600 sensory perception & 27 & $3.35 \times 10^{-15}$ \\
\hline & $\begin{array}{l}\text { GO:0050911 detection of chemical stimulus involved in } \\
\text { sensory perception of smell }\end{array}$ & 25 & $6.47 \times 10^{-15}$ \\
\hline & $\begin{array}{l}\text { GO:0050907 detection of chemical stimulus involved } \\
\text { in sensory perception }\end{array}$ & 25 & $7.79 \times 10^{-15}$ \\
\hline & GO:0009593 detection of chemical stimulus & 25 & $9.98 \times 10^{-15}$ \\
\hline & GO:0007608 sensory perception of smell & 25 & $1.13 \times 10^{-14}$ \\
\hline \multirow[t]{4}{*}{$\mathrm{CC}$} & GO:0016021 integral to membrane & 39 & $8.59 \times 10^{-16}$ \\
\hline & GO:0031224 intrinsic to membrane & 39 & $2.67 \times 10^{-15}$ \\
\hline & GO:0042612 MHC class I protein complex & 3 & 0.010435218 \\
\hline & GO:0042611 MHC protein complex & 3 & 0.015440507 \\
\hline \multirow[t]{2}{*}{$\mathrm{MF}$} & GO:0004984 olfactory receptor activity & 26 & $9.54 \times 10^{-17}$ \\
\hline & GO:0016503 pheromone receptor activity & 3 & 0.0349 \\
\hline
\end{tabular}

GO, Gene Ontology; BP, biological processes; CC, cellular component; MF, molecular function.

identified in femoral head samples of steroid-induced ANFH rats compared with placebo-treated rat samples. Gene Ontology (GO) and pathway enrichment analyses were performed upon these DEGs. An enrichment map was generated to identify overrepresented themes with regard to the enriched GO terms, and a text-mining gene association network associated with femoral necrosis was generated, followed by the construction of a transcriptional regulation network and a protein-protein interaction
(PPI) network. An improved understanding of the molecular mechanisms underlying GC-induced ANFH will likely provide novel targets for diagnostic and therapeutic treatment.

\section{Materials and methods}

Microarray data. The ArrayExpress Archive of Functional Genomics Data (http://www.ebi.ac.uk/arrayexpress) is one 
Table II. The enriched KEGG pathways of upregulated and downregulated genes.

\begin{tabular}{|c|c|c|}
\hline Term & Count & P-value \\
\hline $\begin{array}{l}\text { Upregulated } \\
\text { rno04740:Olfactory } \\
\text { transduction }\end{array}$ & 9 & 0.0073 \\
\hline $\begin{array}{l}\text { Downregulated } \\
\text { rno04740:Olfactory } \\
\text { transduction }\end{array}$ & 19 & $2.09 \times 10^{-08}$ \\
\hline $\begin{array}{l}\text { rno05332:Graft-versus-host } \\
\text { disease }\end{array}$ & 4 & 0.0018 \\
\hline $\begin{array}{l}\text { rno05330:Allograft } \\
\text { rejection }\end{array}$ & 4 & 0.0020 \\
\hline $\begin{array}{l}\text { rno04940:Type I diabetes } \\
\text { mellitus }\end{array}$ & 4 & 0.0029 \\
\hline $\begin{array}{l}\text { rno05320:Autoimmune } \\
\text { thyroid disease }\end{array}$ & 4 & 0.0030 \\
\hline $\begin{array}{l}\text { rno04514:Cell adhesion } \\
\text { molecules (CAMs) }\end{array}$ & 5 & 0.0052 \\
\hline rno05416:Viral myocarditis & 4 & 0.0081 \\
\hline $\begin{array}{l}\text { rno04612:Antigen processing } \\
\text { and presentation }\end{array}$ & 4 & 0.0084 \\
\hline
\end{tabular}

of three major international repositories for functional genomics public data, alongside the Gene Expression Omnibus at NCBI and the DDBJ Omics Archive (11). The mRNA expression profile deposited by Kerachian et al (9), was downloaded from the ArrayExpress database (accession number, E-MEXP-2751; http://www.ebi.ac.uk/ arrayexpress/experiments/E-MEXP-2751/). The platform used was A-AFFY-99-Affymetrix GeneChip Rat Exon 1.0 ST Array [RaEx-1_0-st-v1]. As the original study described (9), glucocorticoid pellets or placebo pellets were administered to age-matched Wistar Kyoto rats for 6 months. Following the 6 month period of the experiment, rats were sacrificed and tissue samples were obtained from the proximal femur, containing the femoral head. In the present study, a dataset consisting of 5 steroid-induced ANFH rat samples (named Steroid_ANFH) and 5 placebo-treated rat samples (named Placebo_no_ANFH) were used in the follow-up analysis.

Data preprocessing and screening of DEGs. The raw data (CEL. format files) were preprocessed in $\mathrm{R}$ using the Bioconductor package, oligo (12). Data were subjected to background correction, quantile normalization, and calculation of expression values using the robust multi-array average algorithm (13). The probe symbols were then transformed into the corresponding gene symbols. When several probes were mapped to one gene, the average value was calculated and represented as the expression levels of this gene. Finally, the gene expression matrix was obtained.

Upregulated and downregulated DEGs were screened in Steroid_ANFH against Placebo_no_ANFH using the linear models for microarray data (limma) package version 2.14.1 (14) in Bioconductor. In addition, P-values from unpaired t-tests (15) in the limma package were calculated to determine the significance of differences between samples. In the present study, genes with $\log _{2}$ fold change $(F C) \geq 0.4$ and $\mathrm{P}<0.05$ were selected as DEGs. Furthermore, the DEGs identified between Steroid_ANFH and Placebo_ no_ANFH were clustered using gplots package (version 2.12.1; https://cran.r-project.org/web/packages/gplots/index. html) (16) in $\mathrm{R}$ to evaluate whether the identified DEGs were sample-specific. The clustering results were visualized as heat maps.

Functional and pathway enrichment analysis. The database for annotation, visualization and integrated discovery (DAVID) is a web-accessible program that provides functional interpretation of large gene lists through facilitating the transition from data collection to biological meaning (17). In the present study, in order to analyze the identified upregulated genes and downregulated genes in Steroid_ANFH on the functional level, GO (18) and Kyoto Encyclopedia of Genes and Genomes (KEGG) (19) pathway enrichment analyses were performed using DAVID (17). The hypergeometric test (20) was applied to calculate $\mathrm{P}$-values. In particular, gene count $\geq 2$ and $\mathrm{P}<0.05$ were selected as the threshold for identifying significant $\mathrm{GO}$ terms and KEGG pathways.

Visualization of $G O$ enrichment analysis. To gain a full view of the probable functions of the upregulated and downregulated genes, an enrichment map was constructed with the Enrichment Map plugin (21) in Cytoscape version 2.8 (22) (http://www.baderlab.org/Software/EnrichmentMap), using the GO enrichment results as the input. The parameters were set as follows: Similarity cutoff, overlap coefficient; cutoff, 0.5; P-value cutoff, 0.005; false discovery rate $\mathrm{Q}$ value cutoff, 0.1. In the Enrichment Map, each node represented a gene set corresponding to an enriched GO term. Node size corresponded to the total number of genes in each GO term. Each weighted line indicated the overlap between two gene sets and the line thickness indicated the number of overlapping genes between nodes.

Generation of association network through text-mining. The Agilent Literature Search plugin (23) was used in conjunction with Cytoscape (22) for text-mining literature with structured queries and for obtaining gene-association networks according to the text-mining results (24). In the present study, in order to further ensure that the upregulated and downregulated genes were biologically associated with ANFH, the Agilent Literature Search tool (23) was used to query published literature associated with the context, and meanwhile construct an association network according to the text-mining results. 'Femoral necrosis' was provided as context terms while the genes from the identified DEG (upregulated and downregulated genes) gene-set were used as search terms. In addition, the species was limited to 'rat'.

Computational searches for transcription factors (TFS). The standard integrated analysis package Genomatix Software Suite version 2.1 (Genomatix GmbH, Munich, Germany; 


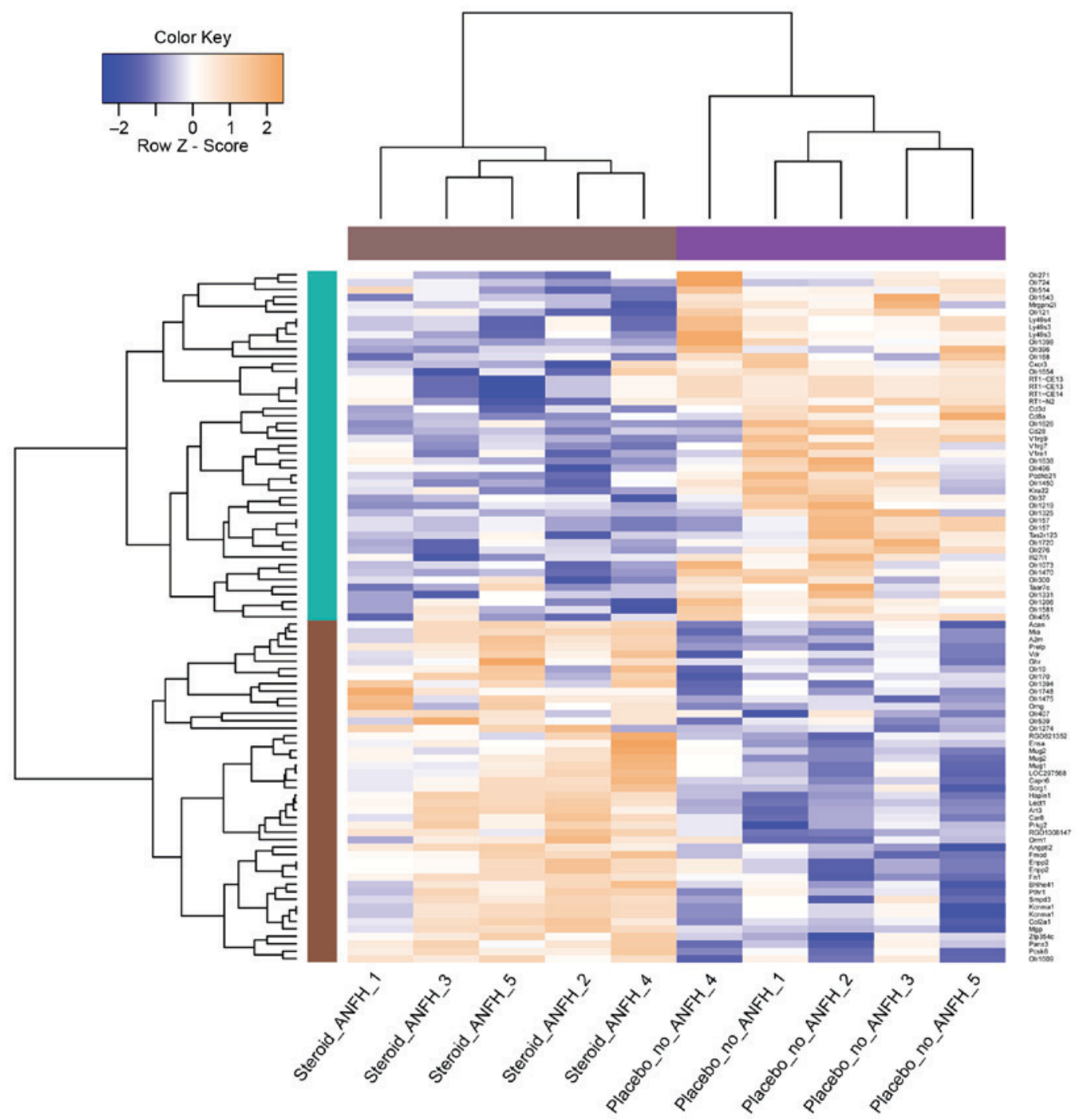

Figure 1. Heat map of differentially expressed genes. Blue or brown indicates higher or lower expression levels of genes, respectively. White indicates genes without altered expression. The brown and purple bars along the top indicate steroid-induced ANFH rat samples and placebo-treated rat samples, respectively. The brown and green bars on the left-hand side indicate the distant boundary of upregulated and downregulated genes.

http://www.genomatix.de) provides large amounts of functional information about genes and their interactions, including TFs (25). In the present study, to gain insights into the transcriptional regulation of the identified DEGs, a software tool within the Genomatix software suite (http:// www.genomatix.de/cgi-bin//GePS/gene-tf_analysis.pl?) (25) was applied to search for IFs that regulated the DEGs and to evaluate the number of TFs with altered expression in the samples. This analysis was based on the literature-derived gene-TF annotation. The input gene-set was the identified upregulated and downregulated genes. The parameter of evidence level was set as the sentence level.

Construction of PPI network. The online database resource Search Tool for the Retrieval of Interacting Genes (STRING) is a comprehensive database providing functional links of proteins, including experimental as well as predicted interaction information by text mining or comparative genomics (26). Interactions of proteins in the STRING database were provided with a confidence score (26). In the present study, a PPI network was constructed using data derived from the STRING database. Genes included in the PPI network were all identified DEGs and a confidence score $\geq 0$.4 was set as the threshold. Proteins encoded by upregulated and downregulated genes were represented by nodes in the network, and lines indicated interactions among proteins. Hub proteins in the PPI network were deemed as those that interacted with the most proteins and that had a higher degree. Cytoscape software version 2.8 (22) was applied for network visualization.

\section{Results}

DEG screening. Compared with Placebo_no_ANFH controls, a total of 46 upregulated genes and 47 downregulated genes were identified in Steroid_ANFH samples. The mean $\log _{2} \mathrm{FC}$ value of upregulated genes was 0.5655 while the average $\log _{2} \mathrm{FC}$ value of downregulated genes was -0.5832 . Furthermore, the expression pattern of identified DEGs correctly distinguished the Steroid_ANFH samples 

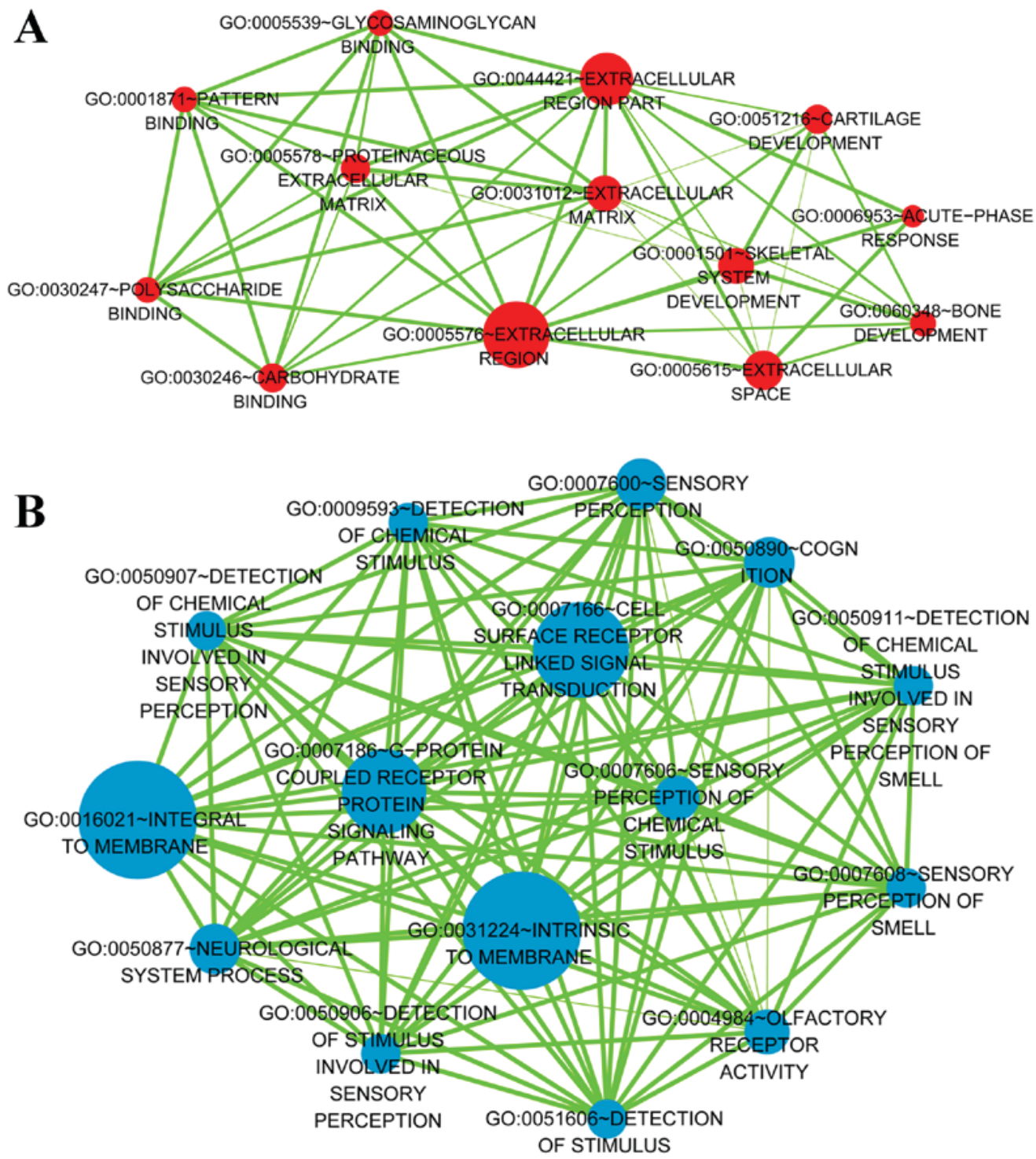

Figure 2. Enrichment map of GO terms of up and downregulated genes. (A) Enrichment map of GO terms of upregulated genes. Red nodes indicate GO terms of upregulated genes. The node size represents the number of genes that belong to this $\mathrm{GO}$ term. The width of the line (green color) is proportional to the size of the overlapping genes. (B) Enrichment map of GO terms of downregulated genes. Blue nodes represent a GO term enriched by downregulated genes and the node size is representative of the number of enriched genes in this GO term. Each weighted line (green color) represents the overlapping genes between the two GO terms.

from Placebo_no_ANFH control samples, as visualized in Fig. 1.

Biological interpretation of ANFH gene expression signature. GO terms, including biological processes, cellular component, and molecular function categories of the significantly up and downregulated genes were summarized in Table I. The results demonstrated that the upregulated genes were significantly concerned with bone development processes, including cartilage development, skeletal system development, bone development and chondrocyte differentiation. The downregulated genes were enriched in different GO terms, including cell surface receptor-linked signal transduction and detection of chemical stimulus. The results of KEGG pathway enrichment analysis were summarized in Table II. From the results, upregulated genes were revealed to be involved in one pathway; olfactory transduction. The downregulated genes were enriched in distinct pathways, including antigen processing and presentation.

Overview of enrichment map profiling. In the present study, the enrichment map was generated to unravel overrepresented themes in ANFH through enrichment analysis with regard to the enriched GO terms. The enrichment maps of upregulated genes and downregulated genes were visualized in Fig. 2A and B, respectively. The enrichment network algorithmically clustered GO terms with highly similar genes and the GO term of GO:0001501 SKELETAL SYSTEM DEVELOPMENT was interconnected with several other GO terms by gene overlap. The degree of this node (GO:0001501) was 7. The enrichment map of downregulated genes revealed that GO terms in this enrichment network were tightly linked and the degree of each GO term in this network was 14 . 


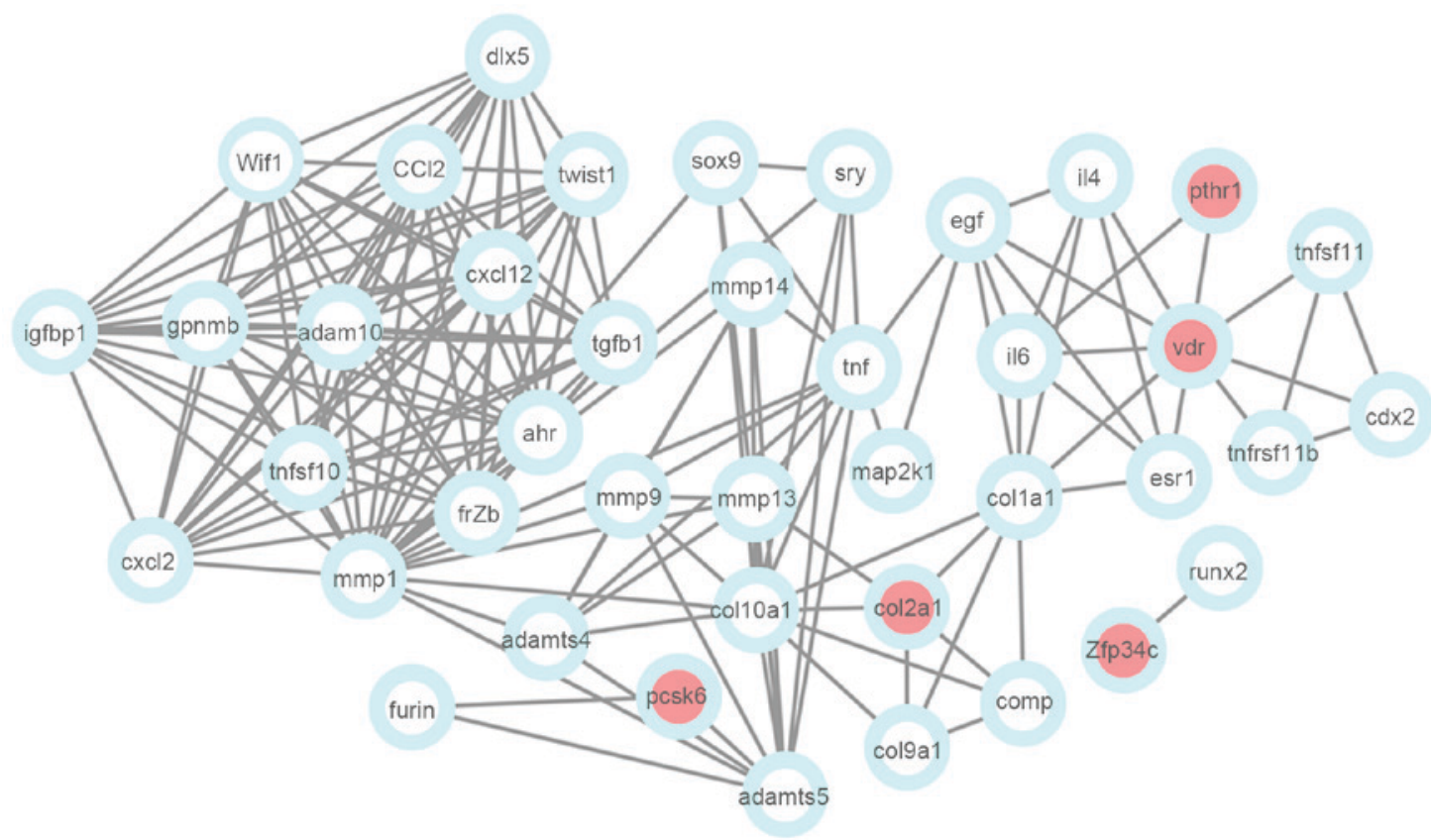

Figure 3. A text-mining association network, associated with femoral necrosis. Red nodes indicate upregulated genes. Each line indicates the interaction between two genes, identified by mining the literature.

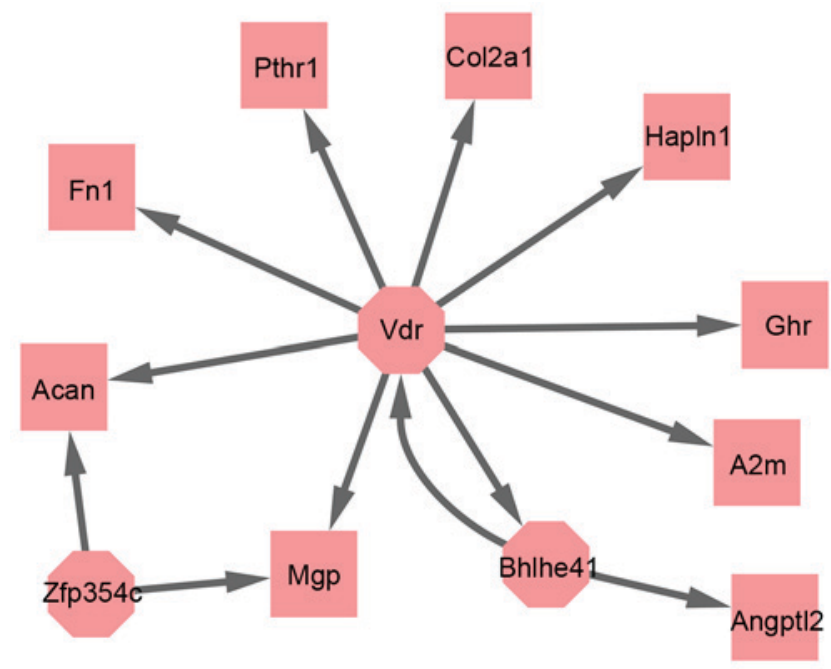

Figure 4. Transcriptional regulation network. Octagonal nodes represent transcription factors. Square nodes represent the target genes.

Extracting significant associations and the construction of the literature mined network of femoral necrosis. With the available data in regard to interactions of different proteins, protein partners for the genes associated with femoral necrosis were searched for using the Agilent Literature Search tool, and a text-mining association network associated with femoral necrosis was generated (Fig. 3). The literature mined network analysis revealed that a total of 5 upregulated genes identified in the present study were involved in the network, including parathyroid hormone receptor 1 (PTHRl), vitamin D (1,25-Dihydroxyvitamin D3) receptor (VDR), collagen, type II, $\alpha 1$ (COL2A1), proprotein convertase subtilisin/kexin type 6 (PCSK60), and zinc finger protein 354C (ZFP354C). These 5 upregulated genes were identified to be associated with femoral necrosis, and interacted with other disease-related genes to generate the network.

The construction of transcriptional regulation network and PPI network. The transcriptional regulation network was visualized in Fig. 4. The results revealed that out of all the DEGs 3 TFs were identified, namely ZFP354C, VDR, and basic helix-loop-helix family, member E41 (BHLHE41). These 3 TFs were all upregulated and their target genes were also upregulated (Fig. 4). In addition, ZFP354C and VDR were also identified as associated with femoral necrosis, as mentioned above.

Furthermore, a PPI network was constructed (Fig. 5). The PPI network consisted of 31 nodes and 67 lines (interactions). The results revealed that the interactive relationships of proteins were relatively simple. However, olfactory receptor genes (including Olr168 and Olr1654) were revealed to make up an interaction module. Furthermore, the PPI network revealed that $P T H R 1$ interacted with $V D R$. A2M was also revealed to interact with fibronectin $1(F N I)$.

\section{Discussion}

There is a considerable negative effect of ANFH on the workforce and, subsequently, on economics. Major efforts are being made to understand GC-induced ANFH at a molecular level. In the present study, a total of 93 DEGs (46 up and 47 downregulated genes) were identified in GC-induced ANFH samples compared with controls. These DEGs were enriched in different GO terms and pathways, including chondrocyte differentiation and detection of chemical stimuli. The enrichment map revealed that the GO term of skeletal system development was interconnected with several other GO terms by gene overlap. The literature mined network analysis revealed that 5 upregulated genes were associated with femoral necrosis, including PTHR1, VDR, COL2A1, 


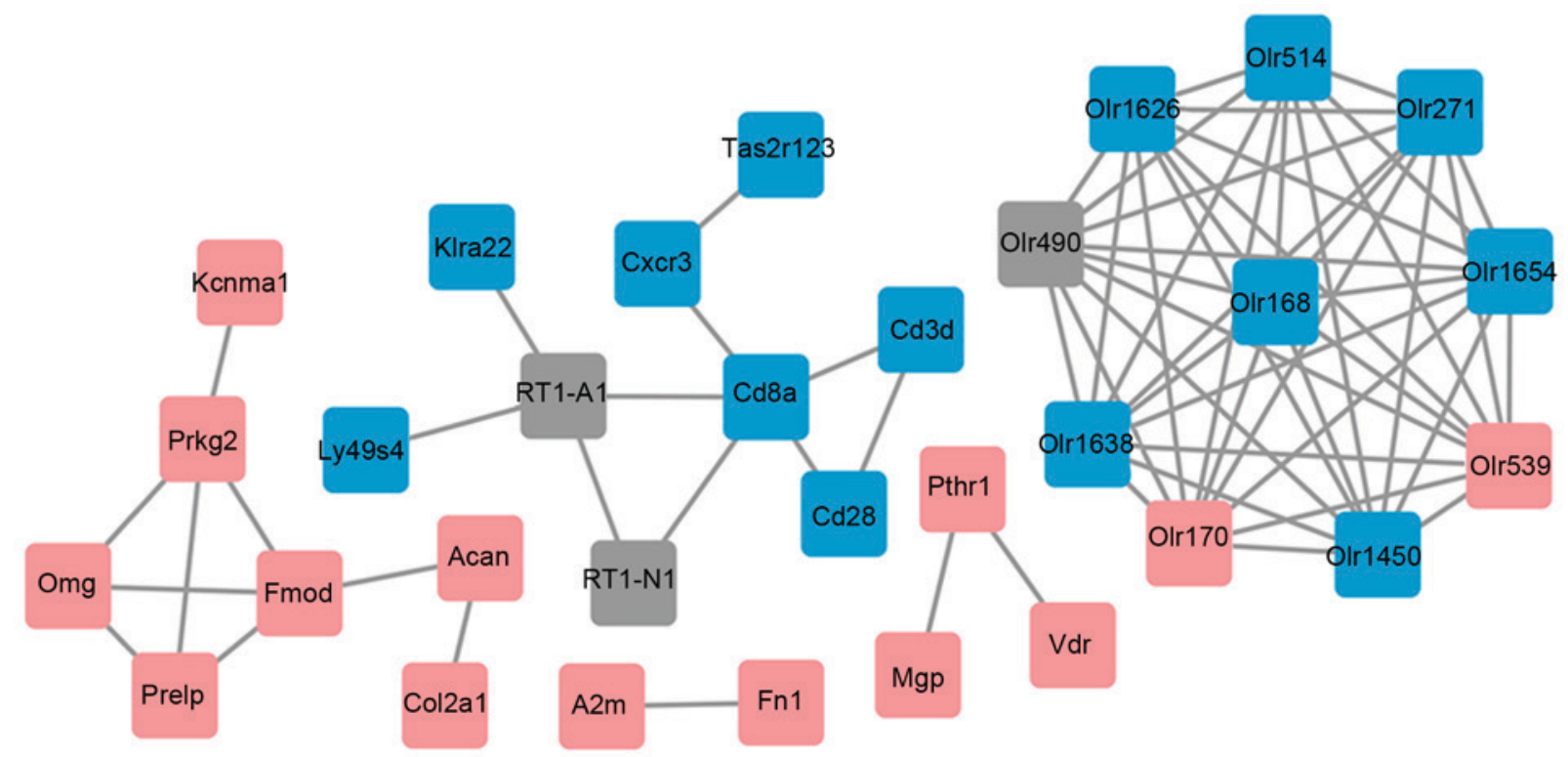

Figure 5. Protein-protein interaction network. Red nodes indicate upregulated genes. Blue nodes represent downregulated genes. Gray nodes indicate genes without altered expression.

PCSK60, and ZFP354C. In addition, ZFP354C and VDR were also identified as TFs. Furthermore, PTHRl was identified to interact with $V D R$ and $A 2 M$ was revealed to interact with $F N 1$ in the PPI network.

The protein encoded by PTHRI is a member of the $\mathrm{G}$-protein coupled receptor family 2 , and this protein is a receptor for parathyroid hormone $(\mathrm{PTH})$ and for parathyroid hormone-like hormone (PTHLH) (27). Previous studies have demonstrated that injected PTH may be used to restore bone loss resulting from excessive glucocorticoid use, and PTH-associated protein peptides may be useful as putative bone regenerative therapies in GC-related bone diseases $(28,29)$. On the other hand, VDR encodes the nuclear hormone receptor for vitamin D3, and VDR may act as a TF that mediates the action of vitamin D3 through controlling the expression of hormone sensitive genes (30). Previous evidence demonstrates that $V D R$ polymorphisms are putatively associated with the risk of osteonecrosis and are significantly associated with the development of osteonecrosis (31). In addition, the work of Goltzman et al (32) revealed that the development of the cartilaginous growth plate and parathyroid gland size were each coordinately regulated by calcium and by the active form of vitamin D, 1,25-dihydroxyvitamin D [1,25 (OH)2D], and that an intact 1,25(OH)2D/VDR system was required for an appropriate osteoclastic response to increased PTH. Consistent with this notion, the present study revealed that the upregulated gene $P T H R 1$ may interact with another upregulated gene, $V D R$, in the PPI network. Collectively, this suggests that PTHR1 may be involved in the pathogenesis of GC-induced ANFH via interacting with $V D R$.

The protein encoded by $A 2 M$ is a protease inhibitor and cytokine transporter (33). A2M inhibits multiple proteases, including trypsin, thrombin and collagenase (33). Kerachian et al (9) reported that A2M shared vascular, cartilaginous and osteogenic functions and may be involved in the development of early GC-induced ANFH (9). FN1 encodes fibronectin, a glycoprotein present on the cell surface and in the extracellular matrix, and this protein is involved in cell adhesion and migration processes (34). Previous data has revealed that fibronectin-aggrecan complex may be useful as a marker for cartilage degradation in patients with non-arthritic hip pain (35). ANFH is characterized by pain in the hip, bone collapse and functional loss (6). In addition, a close connection was identified between $F N 1$ and $A 2 M$ genes in the study of Zhang et al (36). Furthermore, A2M was revealed to interact with $\mathrm{FHI}$ in the PPI network in the present study. In this context, it is possible to suggest that $A 2 M$ has an interaction with $F N 1$ and is involved in the development of GC-induced ANFH. However, further studies and experiments are required to confirm this finding.

However, the present study has several limitations. First, the sample size is small, so further investigations based on larger samples may be required. Second, this is a computational study based on bioinformatics approaches, which gives simulated results. The lack of cross-checking and further experimental verification were also a limitation of the present investigation. Statistical validation using other datasets may be used to cross-check these results. Furthermore, experimental verifications will be performed to determine the findings in future studies using different approaches, including reverse transcription-quantitative polymerase chain reaction and western blot analysis.

In conclusion, the present study demonstrated that several potential key genes ( $P T H R 1, V D R, A 2 M$, and FN1) were associated with the development of GC-induced ANFH. PTHRI may be involved in the pathogenesis of GC-induced ANFH via interacting with $V D R$. $A 2 M$ may also have a significant function in the development of GC-induced ANFH through interacting with FN1. Further investigations with more samples as well as further experimental validation may provide novel insights into the mechanism of GC-induced ANFH and will aid the discovery of novel diagnostic and therapeutic approaches. 


\section{References}

1. Chan KL and Mok CC: Glucocorticoid-induced avascular bone necrosis: Diagnosis and management. Open Orthop J 6: 449-457, 2012.

2. Weinstein RS: Clinical practice. Glucocorticoid-induced bone disease. N Engl J Med 365: 62-70, 2011.

3. Colomb-Lippa D: Avascular necrosis of the femoral head. JAAPA 27: 40-41, 2014

4. Weinstein RS: Glucocorticoid-induced osteoporosis and osteonecrosis. Endocrinol Metab Clin North Am 41: 595-611, 2012

5. Kerachian MA, Séguin C and Harvey EJ: Glucocorticoids in osteonecrosis of the femoral head: A new understanding of the mechanisms of action. J Steroid Biochem Mol Biol 114: 121-128, 2009.

6. Bekler H, Uygur AM, Gökçe A and Beyzadeoğlu T: The effect of steroid use on the pathogenesis of avascular necrosis of the femoral head: An animal model. Acta Orthop Traumatol Turc 41: 58-63, 2007 (In Turkish).

7. Bian Y, Qian W, Li H, Zhao RC, Shan WX and Weng X: Pathogenesis of glucocorticoid-induced avascular necrosis: A microarray analysis of gene expression in vitro. Int $\mathrm{J}$ Mol Med 36: 678-684, 2015

8. Zalavras C, Shah S, Birnbaum MJ and Frenkel B: Role of apoptosis in glucocorticoid-induced osteoporosis and osteonecrosis. Crit Rev Eukaryot Gene Expr 13: 221-235, 2003.

9. Kerachian MA, Cournoyer D, Harvey EJ, Chow TY, Bégin LR, Nahal A and Séguin C: Research article New insights into the pathogenesis of glucocorticoid-induced avascular necrosis: microarray analysis of gene expression in a rat model. 2010.

10. Samara S, Dailiana Z, Chassanidis C, Koromila T, Papatheodorou L, Malizos KN and Kollia P: Expression profile of osteoprotegerin, RANK and RANKL genes in the femoral head of patients with avascular necrosis. Exp Mol Pathol 96 : 9-14, 2014.

11. Rustici G, Kolesnikov N, Brandizi M, Burdett T, Dylag M, Emam I, Farne A, Hastings E, Ison J, Keays M, et al: Arrayexpress update-trends in database growth and links to data analysis tools. Nucleic Acids Res 41 (Database Issue): D987-D990, 2013.

12. Carvalho BS and Irizarry RA: A framework for oligonucleotide microarray preprocessing. Bioinformatics 26: 2363-2367, 2010.

13. Silver JD, Ritchie ME and Smyth GK: Microarray background correction: Maximum likelihood estimation for the normal-exponential convolution. Biostatistics 10: 352-363, 2009.

14. Smyth GK: Limma: linear models for microarray data. In: Bioinformatics and computational biology solutions using R and Bioconductor. Springer: pp397-420, 2005.

15. Cindrova-Davies T, Yung HW, Johns J, Spasic-Boskovic O, Korolchuk S, Jauniaux E, Burton GJ and Charnock-Jones DS: Oxidative stress, gene expression, and protein changes induced in the human placenta during labor. Am J Pathol 171: 1168-1179, 2007.

16. Zhang Y, Limaye PB, Renaud HJ and Klaassen CD: Effect of various antibiotics on modulation of intestinal microbiota and bile acid profile in mice. Toxicol Appl Pharmacol 277: 138-145, 2014.

17. Dennis G Jr, Sherman BT, Hosack DA, Yang J, Gao W, Lane HC and Lempicki RA: DAVID: Database for annotation, visualization and integrated discovery. Genome Biol 4: P3, 2003.

18. Ashburner M, Ball CA, Blake JA, Botstein D, Butler $\mathrm{H}$ Cherry JM, Davis AP, Dolinski K, Dwight SS, Eppig JT, et al: Gene ontology: Tool for the unification of biology. The gene ontology consortium. Nat Genet 25: 25-29, 2000.

19. Kanehisa M, Sato Y, Kawashima M, Furumichi M and Tanabe M: KEGG as a reference resource for gene and protein annotation. Nucleic Acids Res 44: D457-D462, 2016.
20. Mao X, Cai T, Olyarchuk JG and Wei L: Automated genome annotation and pathway identification using the KEGG Orthology (KO) as a controlled vocabulary. Bioinformatics 21: 3787-3793, 2005 .

21. Merico D, Isserlin R, Stueker O, Emili A and Bader GD: Enrichment map: A network-based method for gene-set enrichment visualization and interpretation. PLoS One 5: e13984, 2010.

22. Smoot ME, Ono K, Ruscheinski J, Wang PL and Ideker T: Cytoscape 2.8: New features for data integration and network visualization. Bioinformatics 27: 431-432, 2011.

23. Cline MS, Smoot M, Cerami E, Kuchinsky A, Landys N, Workman C, Christmas R, Avila-Campilo I, Creech M, Gross B, et al: Integration of biological networks and gene expression data using Cytoscape. Nat Protoc 2: 2366-2382, 2007.

24. Priyadarshini S and Aich P: Effects of psychological stress on innate immunity and metabolism in humans: A systematic analysis. PLoS One 7: e43a232, 2012.

25. Werner T, Dombrowski SM, Zgheib C, Zouein FA, Keen HL, Kurdi M and Booz GW: Elucidating functional context within microarray data by integrated transcription factor-focused gene-interaction and regulatory network analysis. Eur Cytokine Netw 24: 75-90, 2013.

26. Franceschini A, Szklarczyk D, Frankild S, Kuhn M, Simonovic M, Roth A, Lin J, Minguez P, Bork P, von Mering C and Jensen LJ: String v9. 1: Protein-protein interaction networks, with increased coverage and integration. Nucleic Acids Res 41 (Database issue): D808-D815, 2013.

27. Tenne M, McGuigan F, Jansson L, Gerdhem P, Obrant KJ, Luthman $\mathrm{H}$ and Akesson $\mathrm{K}$ : Genetic variation in the PTH pathway and bone phenotypes in elderly women: Evaluation of PTH, PTHLH, PTHR1 and PTHR2 genes. Bone 42: 719-727, 2008.

28. de Castro LF, Lozano D, Dapía S, Portal-Núñez S, Caeiro JR, Gómez-Barrena E and Esbrit P: Role of the N-and C-terminal fragments of parathyroid-hormone-related protein as putative therapies to improve bone regeneration under high glucocorticoid treatment. Tissue Eng Part A 16: 1157-1168, 2010.

29. Morley P, Whitfield JF and Willick GE: Therapeutic prospects for parathyroid hormone and parathyroid hormone analogs. Current Medicinal Chemistry-Immunology, Endocrine \& Metabolic Agents 1: 269-287, 2001.

30. Carlberg C and Campbell MJ: Vitamin D receptor signaling mechanisms: Integrated actions of a well-defined transcription factor. Steroids 78: 127-136, 2013.

31. Relling MV, Yang W, Das S, Cook EH, Rosner GL, Neel M, Howard S, Ribeiro R, Sandlund JT, Pui CH and Kaste SC: Pharmacogenetic risk factors for osteonecrosis of the hip among children with leukemia. J Clin Oncol 22: 3930-3936, 2004.

32. Goltzman D: Emerging roles for calcium-regulating hormones beyond osteolysis. Trends in Endocrinology \& Metabolism 21: 512-518, 2010.

33. Rehman AA, Ahsan H and Khan FH: $\alpha-2-$ Macroglobulin: A physiological guardian. J Cell Physiol 228: 1665-1675, 2013.

34. Martino MM, Tortelli F, Mochizuki M, Traub S, Ben-David D, Kuhn GA, Müller R, Livne E, Eming SA and Hubbell JA: Engineering the growth factor microenvironment with fibronectin domains to promote wound and bone tissue healing. Sci Transl Med 3: 100ra89, 2011

35. Abrams GD, Safran MR, Shapiro LM, Maloney WJ, Goodman SB, Huddleston JI, Bellino MJ and Scuderi GJ: Fibronectin-aggrecan complex as a marker for cartilage degradation in non-arthritic hips. Knee Surg Sports Traumatol Arthrosc 22: 768-773, 2014.

36. Zhang P, Li Y, Zhang LD, Wang LH, Wang X, He C and Lin ZF: Proteome changes in mesenteric lymph induced by sepsis. Mol Med Rep 10: 2793-2804, 2014. 